\title{
Pose Adjustment Capability of Secondary Mirror in Correcting Gravity Deformation of Large Aperture Remote Sensor
}

\author{
Xianbin $\mathrm{Li}^{1,2^{*}}$, Jiang $\mathrm{Guo}^{1}$, Mingdong Shao ${ }^{1}$, Yuanpeng $\mathrm{Li}^{1}$, Qingwen $\mathrm{Wu}^{1}$ \\ ${ }^{1}$ Changchun Institute of Optics, Fine Mechanics and Physics, Chinese Academy of Sciences, Changchun 130033, China \\ ${ }^{2}$ University of Chinese Academy of Sciences, Beijing 100049, China
}

Corresponding Author Email: 1xb51@126.com

https://doi.org/10.18280/i2m.190211

Received: 17 October 2019

Accepted: 2 January 2020

\section{Keywords:}

large aperture, gravity deformation, misalignment-induced aberration, secondary mirror, pose adjustment

\begin{abstract}
For large aperture remote sensor, it is a difficult task to ensure the ultrahigh pose accuracy of mirrors. To solve the problem, this paper attempts to correct the pose misalignment induced by the gravity deformation through pose adjustment of the secondary mirror. Firstly, an ultra-lightweight remote sensor (diameter: $2.4 \mathrm{~m}$ ) was designed, and a finite element model (FEM) was constructed to analyze the influence of gravity deformation on the sensor. The analysis shows that the gravity deformation of the primary mirror and the secondary mirror exceeded the tolerance limits. Hence, a pose adjustment design was proposed for the secondary mirror to correct the misalignment of the optical system. To verify its correction effect, the pose adjustment plan was applied to correct the misalignments of the optical system caused by gravity deformation. The results show that the pose adjustment plan eliminated the effect of gravity on the ground, and corrected the misalignments after the sensor is in orbit. Overall, the proposed design greatly reduces the fabrication difficulty of the large aperture remote sensor, and effectively improves the sensor's imaging quality in orbit.
\end{abstract}

\section{INTRODUCTION}

Long focal length and large aperture are two defining trends of remote sensing technology [1-4]. Large aperture remote sensor must satisfy strict performance requirements in orbit. Once the remote sensor is in orbit, the elements of the optical system must maintain ultrahigh pose accuracy, such that the wave aberration of the optical system meets the imaging requirements [5-7].

The remote sensor orbits the earth and works in a microgravity environment. However, the fabrication process of the remote sensor on the ground is inevitably affected by gravity deformation. The influence of the gravity on the opticalmechanical structure increases with the aperture and focal length of remote sensor, making it increasingly difficult to ensure the pose accuracy of the mirrors [8]. The negative effect of the gravity on the remote sensor during the fabrication and detection will severely undermine the pose accuracy of mirrors in large aperture, long focal length optical system in orbit.

Furthermore, the gravity deformation of the mirrors, their supports, and fuselage is included in the optical system, when the remote sensor is finetuned to the optimal state. After the remote sensor enters the orbit, gravity deformation will be released in the micro-gravity environment, resulting in minor pose misalignment to the mirrors of the optical system. The ensuing misalignment of the optical system will affect the inorbit imaging quality $[9,10]$.

Traditionally, the misalignment-induced aberration is solved through structural optimization and rigidity design. There is a precondition for the traditional method: the gravity deformation of the large aperture remote sensor should not surpass the requirements on optical performance. In other words, large diameter mirrors and their supports must be rigid enough, and the gravity deformation of fuselage must be sufficiently small. Hence, the traditional method seriously limits the lightweight design of large aperture remote sensor. The Hubble Space Telescope is a typical example of the traditional design: the weights of the primary mirror and the whole machine are $828 \mathrm{~kg}$ and $11,110 \mathrm{~kg}$, respectively.

Recently, some new ideas have emerged to solve the gravity deformation of large aperture remote sensor, namely, the ground gravity unloading technology. By this technology, a special auxiliary unloading mechanism is added to the remote sensor, which optimizes the unloading position and the magnitude of unloading force. In this way, the negative effect of gravity on large aperture optical system is effectively reduced $[11,12]$.

The surface accuracy of large diameter mirror is greatly affected by gravity. It is impossible to ensure that the surface accuracy meets the requirements of optical design, for solely relying on the positioning support structure of the mirror. This calls for gravity compensation on the mirror during ground detection. In general, the effect of gravity can be offset by multi-point auxiliary support [13]. For large aperture remote sensor, the gravity unloading mechanism should be integrated into the sensor design: the unloading position should be determined in the design of opto-mechanical structure, and the transfer path of the unloading force should be specified in the fuselage design. But the integrated design only guarantees that the unloading force will not induce large local stress or affect the optical performance of the remote sensor [14].

The existing studies on space instruments and gravity unloading mostly focus on highly rigid precision instruments, such as robotic arms and satellite platforms. Large aperture 
remote sensor is generally of low rigidity, due to its limited size and weight, and thus sensitive to local stress. Thus, it is very difficult to design or implement a suitable gravity unloading mechanism for large aperture remote sensor. To make matters worse, the implementation effect might deviate from the results of simulation analysis, which affects the effect of gravity unloading.

To ensure the in-orbit performance of remote sensor, the gravity unloading mechanism should provide an unloading force of highly precise magnitude and direction. The layout of unloading points must be designed carefully according to the deformation mechanism and structural configuration of the remote sensor. In addition, the remote sensor should be rigid enough to offer a transfer path of the unloading force, in order to control the stress induced by the unloading force.

Focusing on the ultra-lightweight large aperture remote sensor, this paper attempts to correct the misalignmentinduced aberration of the optical system through pose adjustment of the secondary mirror, eliminating the negative effect of gravity that cannot be effectively mitigated by gravity unloading methods. Firstly, the misalignment features of the optical system were analyzed in details. Next, the effect of gravity on the target sensor was simulated, and the gravity deformation was quantified. On this basis, a pose adjustment mechanism was designed for the secondary mirror of the target sensor. Finally, the proposed mechanism was proved effective in correcting the misalignment-induced aberration of the target sensor both on the ground and in the orbit

\section{MISALIGNMENT FEATURES OF OPTICAL SYSTEM}

\subsection{Optical system design}

Most large aperture remote sensors adopt coaxial optical system, which is more compact than off-axis system in the direction vertical to the optical axis. For example, the Hubble Space Telescope uses the Cassegrain system. The latest design of coaxial optical system tends to include multiple mirrors.

As shown in Figure 1, this paper designs a three-mirror anastigmat (TMA) optical system with an offset field-of-view (FOV). The diameter, f-number $(F \#)$, and FOV of the proposed system are $2.4 \mathrm{~m}, 15$, and $0.4^{\circ} \times 0.2^{\circ}$, respectively. The central FOV is offset by $0.25^{\circ}$ in the $\mathrm{X}$ direction, as shown in Figure 2. The residual aberration of the entire FOV peaks at $0.022 \lambda(\lambda=632.8 \mathrm{~nm})$.

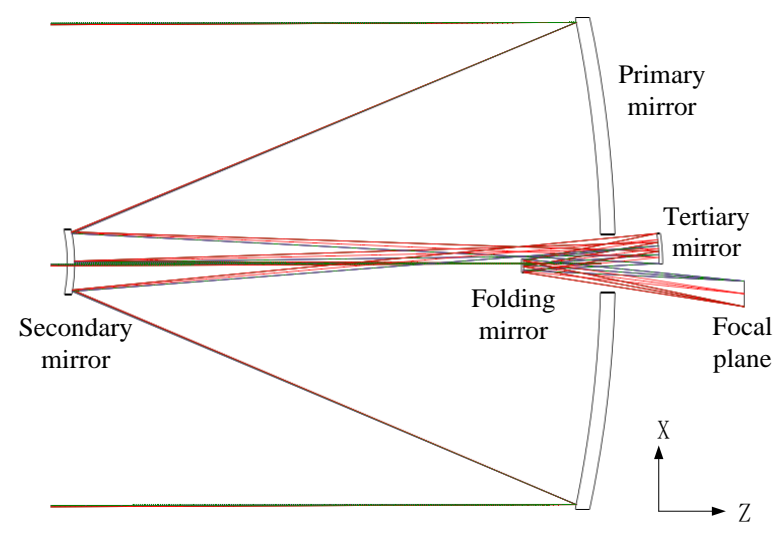

Figure 1. Design of the optical system

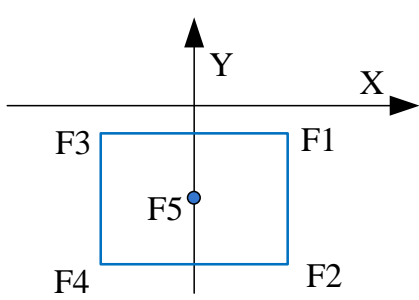

Figure 2. FOVs of the optical system

In the proposed optical system, each mirror has 6 degrees of freedom (DOFs): the deviations in the $\mathrm{X}, \mathrm{Y}$, and $\mathrm{Z}$ directions (Dx, Dy, Dz), and the deflections around the $\mathrm{X}, \mathrm{Y}$, and $\mathrm{Z}$ axes (Tx, Ty, Tz). Among the six DOFs, the Dz of the primary and secondary mirrors will not undermine the rotational symmetry of the optical system, but introduce spherical aberration and defocus aberration. Because of rotational symmetry of the mirrors, the Tz will not induce any aberration. Therefore, five DOFs, namely, Dx, Dy, Dz, Tx, and Ty, are mainly analyzed here for the primary, secondary, and tertiary mirrors.

\subsection{Misalignment model of the optical system}

According to the basic theory of aberration, the wave aberration induced by misaligned mirrors in the optical system is a function of the pose misalignment of each mirror:

$$
W(\rho, \theta)=f(U)
$$

where, $W(\rho, \theta)$ is the wave front aberration in the $\rho-\theta$ polar coordinate system; $U=\left(x_{1}, x_{2}, \ldots, x_{n}\right)$ is the set of pose misalignments of each mirror.

However, it is difficult to establish a functional relationship between aberration of the optical system and the pose misalignments of each mirror. The misalignment-induced aberration of the optical system is often fitted by ZFR, using its orthogonality. But the functional relationship between the Zernike coefficient and the misalignment is too complicated to obtain easily.

According to Taylor's theorem for multivariate functions, a multivariate function $f(x)$ can be expanded into a Taylor series, if it is continuous in the neighborhood of point $x_{0}$ and has $(\mathrm{n}+$ 1)-order continuous derivatives. Taking only the first-order approximation, the Zernike coefficient can be established by the approximate relationship with misalignment:

$$
\begin{aligned}
& {\left[\begin{array}{c}
Z_{1}^{(1)}(N+\Delta N) \\
\vdots \\
Z_{i}^{(j)}(N+\Delta N) \\
\vdots
\end{array}\right]=\left[\begin{array}{c}
Z_{1}^{(1)}(N) \\
\vdots \\
Z_{i}^{(j)}(N) \\
\vdots
\end{array}\right]} \\
& +\left[\begin{array}{cccc}
\frac{\partial Z_{1}^{(1)}(N)}{\partial x_{1}} & \frac{\partial Z_{1}^{(1)}(N)}{\partial x_{2}} & \ldots & \frac{\partial Z_{1}^{(1)}(N)}{\partial x_{n}} \\
\vdots & \vdots & \vdots & \vdots \\
\frac{\partial Z_{i}^{(j)}(N)}{\partial x_{1}} & \frac{\partial Z_{i}^{(j)}(N)}{\partial x_{2}} & \ldots & \frac{\partial Z_{i}^{(j)}(N)}{\partial x_{n}} \\
\vdots & \vdots & \vdots & \vdots
\end{array}\right]\left[\begin{array}{c}
\Delta x_{1} \\
\Delta x_{2} \\
\vdots \\
\Delta x_{n}
\end{array}\right]
\end{aligned}
$$

It can be seen that misalignment-induced aberration is the product of the partial derivative of the Zernike coefficient on the pose of the optical element and the pose misalignment of 
the optical element. Thus, the partial derivative of the Zernike coefficient on the pose misalignment is the coefficient between aberration and misalignment. The partial derivation describes how sensitive the aberration is to misalignment.

In optical inspection, the wave aberration is often illustrated by Fringe Zernike polynomials (ZFR). The coefficients of ZFR can depict different types of basic aberrations of optical system. However, these coefficients are difficult to analyze, and the pose misalignment is limited of each mirror. Hence, the linear equation between Zernike coefficient and misalignment can be obtained based on differential quotient rather than differential, laying the basis for modelling the misalignment correction of the optical system.

According to the relationship between the Zernike polynomial coefficient and the misalignment, the aberration of the optical system induced by its adjustable variables $x_{i}$ can be expressed as:

$$
\triangle Z=A \cdot \triangle X
$$

In order to compensate for the misalignment-induced aberration, the variables should be adjusted by the following amount:

$$
\triangle X=A^{-1} \cdot \triangle Z
$$

where, $A^{-1}$ is the sensitivity matrix of the optical system. The matrix, as an attribute of the optical system, can be established based on the design parameters of the system.

After the adjustable variables of the optical system are selected, the adjustment amount of the variables can be solved through the above method based on the misalignment-induced aberration. If there are fewer adjustable variables than the type of misaligned elements, the adjustment amount can be computed by the least squares (LS) method.

\subsection{Misalignment sensitivity of optical system}

The large aperture and long focal length of the optical system severely restrict the size and weight of the remote sensor. This gives birth to ultra-lightweight opticalmechanical structure. The ultra-lightweight design makes the optical system more sensitive to gravity and temperature. Therefore, it is necessary to analyze the requirements of the optical system on pose accuracy of each mirror.

According to the misalignment correction model, the sensitivity matrix reflects how sensitive the misalignmentinduced aberration is to the pose misalignment of each mirror. Thus, it is very meaningful to examine the misalignment sensitivity of the optical system. With the aid of the sensitivity matrix, the pose parameters that are sensitive to system performance can be identified through sensitivity analysis. These parameters should be optimized in the design of opticalmechanical structure, and focused on during the fabrication and detection of the remote sensor $[15,16]$.

In the optical design software Code $\mathrm{V}$, the misalignment sensitivity analysis was performed through single factor analysis. The misalignments of the three mirrors were simulated separately in the optical analysis model.

The simulation results show that the misalignment-induced aberrations of all FOVs changed similarly with the misalignment of the primary mirror. Therefore, only the simulation results of the central FOV F5 are presented here.
Figure 3 shows the variation of Zernike coefficient with misalignment of the primary mirror.
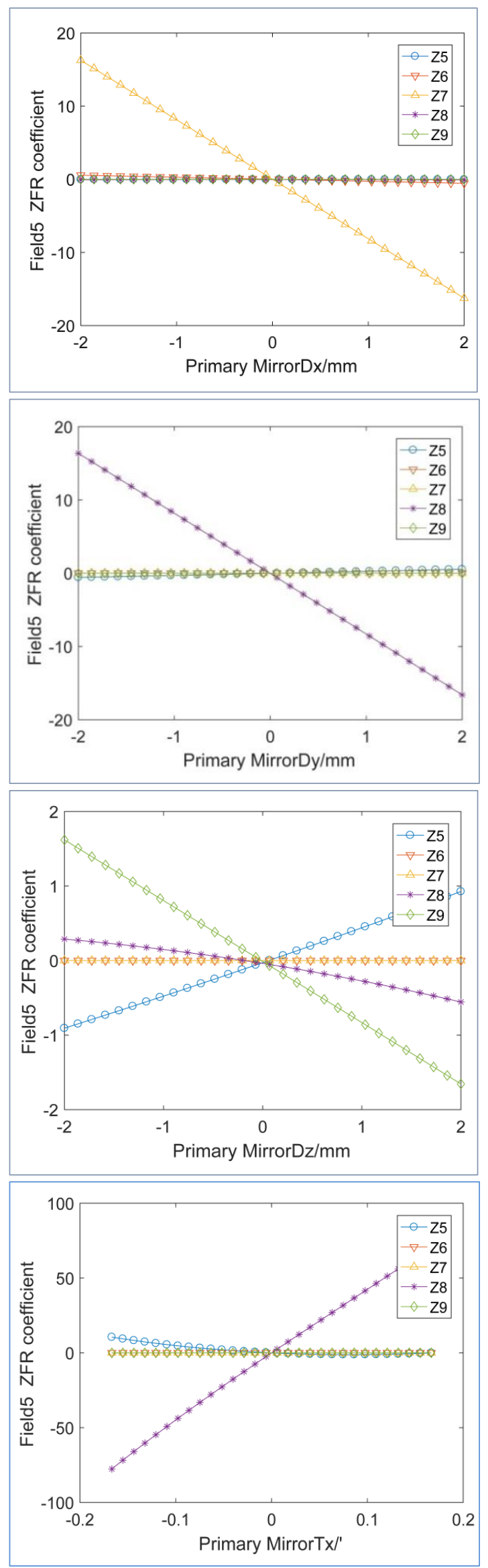


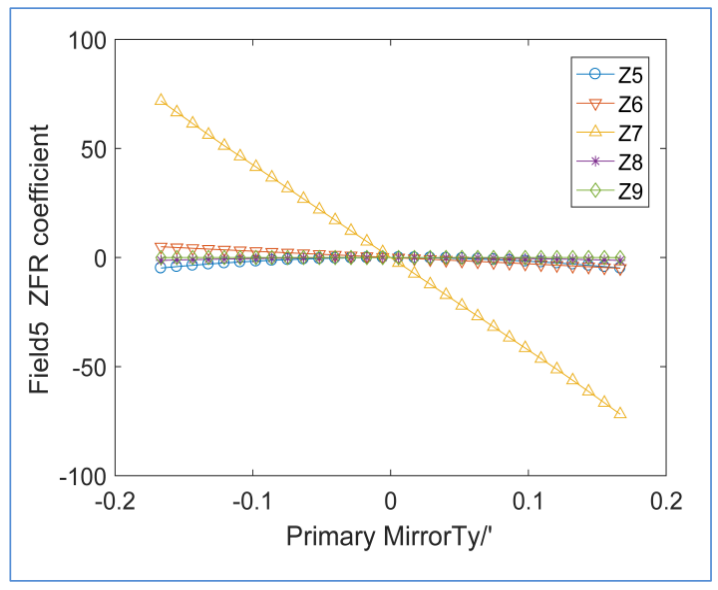

Figure 3. Variation curve of wave aberration ZFR coefficient with the misalignment of the primary mirror in the field of view F5

As shown in Figure 3, the Zernike coefficient of aberration nearly changed linearly with the misalignment of the primary mirror, when the primary mirror deviated $\pm 2 \mathrm{~mm}$ in position and deflected $\pm 0.2^{\prime}$ in angle. The comatic aberration (corresponding to Z7 and Z8) was particularly sensitive to this misalignment. Simulation analysis shows that the aberration of the central FOV F5 also changed almost linearly with the misalignments of the secondary and tertiary mirrors. This means it is feasible to establish the relationship between mirror misalignment and system aberration based on the sensitivity matrix.

Next, the central FOV F5 was selected as an example of the optical system for the analysis of misalignment sensitivity. The results of the sensitivity analysis are listed in Tables 1-3, where $\mathrm{Dx}$, Dy and $\mathrm{Dz}$ are the variations in the Zernike coefficient of the aberration induced by $1 \mathrm{~mm}$ deviation in $X$, $\mathrm{Y}$, and $\mathrm{Z}$ directions (unit: $\lambda / \mathrm{mm}$ ); Tx and Ty are the variations in the 5th to 9th ZFR coefficients induced by to 1 ' deflection about the $\mathrm{X}$ and $\mathrm{Y}$ axes (unit: $\lambda /{ }^{\prime}$ ).

As shown in Tables 1-3, the optical system was very sensitive to the Dx, Dy, Tx and Ty offsets of the primary mirror, and very sensitive to the Dx, Dy, Tx and Ty offsets of the secondary mirror, especially to the misalignments of pose angle. By contrastive, the optical system was not highly sensitive to misalignments of the tertiary mirror.

For a diffraction-limited optical system, when the rootmean-square (RMS) of wave aberration is better than $\lambda / 14$, the imaging performance could reach the diffraction limit. Under this requirement, the pose tolerance limit of each mirror in the optical system was obtained through weighted tolerance allocation, according to the results of sensitivity analysis, as well as the development difficulty and cost of each mirror. Table 4 lists the pose tolerance limits of the mirrors in the proposed optical system.

Table 1. Results of sensitivity analysis on pose alignment of the primary mirror

\begin{tabular}{cccccc}
\hline \multirow{2}{*}{ Offset type } & \multicolumn{5}{c}{ Sensitivity coefficient } \\
\cline { 2 - 6 } & $\mathbf{Z 5}$ & $\mathbf{Z 6}$ & $\mathbf{Z 7}$ & $\mathbf{Z 8}$ & $\mathbf{Z 9}$ \\
\hline $\operatorname{Dx} /(\lambda / \mathrm{mm})$ & 0 & -0.2757 & -8.1305 & 0 & 0 \\
$\operatorname{Dy}(\lambda / \mathrm{mm})$ & 0.2793 & 0 & 0 & -8.2436 & -0.0026 \\
$\operatorname{Dz}(\lambda / \mathrm{mm})$ & 0.4585 & 0 & 0 & -0.211 & -0.8185 \\
$\operatorname{Tx}\left(\lambda /{ }^{\prime}\right)$ & -31.7057 & 0 & 0 & 445.6384 & 0.2563 \\
$\operatorname{Ty}\left(\lambda /{ }^{\prime}\right)$ & 0 & -29.6694 & -431.013 & 0 & 0 \\
\hline
\end{tabular}

Table 2. Results of sensitivity analysis on pose alignment of the secondary mirror

\begin{tabular}{cccccc}
\hline \multirow{2}{*}{ Offset type } & \multicolumn{5}{c}{ Sensitivity coefficient } \\
\cline { 2 - 6 } & $\mathbf{Z 5}$ & $\mathbf{Z 6}$ & $\mathbf{Z 7}$ & $\mathbf{Z 8}$ & $\mathbf{Z 9}$ \\
\hline $\operatorname{Dx}(\lambda / \mathrm{mm})$ & 0 & 0.278 & 8.05 & 0 & 0 \\
$\operatorname{Dy}(\lambda / \mathrm{mm})$ & -0.2771 & 0 & 0 & 8.1594 & 0.0029 \\
$\operatorname{Dz}(\lambda / \mathrm{mm})$ & -0.4687 & 0 & 0 & 0.2309 & 0.8208 \\
$\operatorname{Tx}\left(\lambda /{ }^{\prime}\right)$ & 21.5656 & 0 & 0 & -60.3623 & -0.0048 \\
$\operatorname{Ty}\left(\lambda /{ }^{\prime}\right)$ & 0 & 21.3609 & 59.7203 & 0 & 0 \\
\hline
\end{tabular}

Table 3. Results of sensitivity analysis on pose alignment of the tertiary mirror

\begin{tabular}{cccccc}
\hline \multirow{2}{*}{ Offset type } & \multicolumn{5}{c}{ Sensitivity coefficient } \\
\cline { 2 - 6 } & $\mathbf{Z 5}$ & $\mathbf{Z 6}$ & $\mathbf{Z 7}$ & $\mathbf{Z 8}$ & $\mathbf{Z 9}$ \\
\hline $\operatorname{Dx}(\lambda / \mathrm{mm})$ & 0 & -0.0024 & 0.0826 & 0 & 0 \\
$\operatorname{Dy}(\lambda / \mathrm{mm})$ & -0.002 & 0 & 0 & 0.0826 & -0.0003 \\
$\operatorname{Dz}(\lambda / \mathrm{mm})$ & 0.0102 & 0 & 0 & -0.0197 & -0.0023 \\
$\operatorname{Tx}\left(\lambda /^{\prime}\right)$ & -4.9966 & 0 & 0 & -0.5647 & 0.0036 \\
$\operatorname{Ty}\left(\lambda{ }^{\prime}\right)$ & 0 & -4.9929 & 0.5736 & 0 & 0 \\
\hline
\end{tabular}

Table 4. Pose tolerance limits of each mirror in the optical system

\begin{tabular}{cccc}
\hline Pose tolerance & Primary mirror & Secondary mirror & Tertiary mirror \\
\hline X-direction position deviation & $0.023 \mathrm{~mm}$ & $0.024 \mathrm{~mm}$ & $2.3 \mathrm{~mm}$ \\
Y-direction position deviation & $0.023 \mathrm{~mm}$ & $0.025 \mathrm{~mm}$ & $2.5 \mathrm{~mm}$ \\
Z-direction position deviation & $0.12 \mathrm{~mm}$ & $0.15 \mathrm{~mm}$ & $5.5 \mathrm{~mm}$ \\
Angular deflection around X axis & $1.7^{\prime \prime}$ & $2.8^{\prime \prime}$ & $90^{\prime \prime}$ \\
Angular deflection around Y axis & $1.6^{\prime \prime}$ & $2.8^{\prime \prime}$ & $78^{\prime \prime}$ \\
\hline
\end{tabular}




\section{GRAVITY DEFORMATION ANALYSIS}

\subsection{Ultra-lightweight design and finite-element model (FEM)}

The large aperture primary mirror is the largest and heaviest element of the optical system. Therefore, gravity has greater impacts on this mirror than on any other element. To achieve the purpose of ultra-lightweight design, the $2.4 \mathrm{~m}$-diameter primary mirror was made of reaction bonded (RB) $\mathrm{SiC}$, with a triangular hole on the backside. The weight of the primary mirror was controlled at $265 \mathrm{~kg}$. The primary mirror was supported by a three-point passive structure (Figure 4). The above design optimizes the surface accuracy of the primary mirror under the horizontal optical axis, and minimizes the gravity deformation of the mirror. Through iterative optimization, the surface RSME of the primary error was reduced to $12.24 \mathrm{~nm}$ under $\mathrm{X}$-direction gravity, which is better than $1 / 50 \lambda(\lambda=632.8 \mathrm{~nm})$.

According to the optical design, the proposed remote sensor is mainly composed of a primary mirror assembly, a secondary mirror assembly, a tertiary mirror assembly, a folding mirror assembly, and a fuselage assembly. The ultra-lightweight design should cover the opto-mechanical structure, all the mirrors, and the fuselage, so that the sensor meets the strict requirements on size and weight.

In the optical design, the axial distance between primary and tertiary mirrors is very small. Thus, the two mirrors were arranged on the same bearing frame. Under this arrangement, common reference adjustment could be achieved, and the pose accuracy of the two mirrors could be guaranteed by the stiffness of the main bearing frame.

Meanwhile, the axial distance between secondary and primary mirrors is very large. To control the sensor weight, the support of the secondary mirror was designed as a lightweight space truss of carbon fiber spider structure.

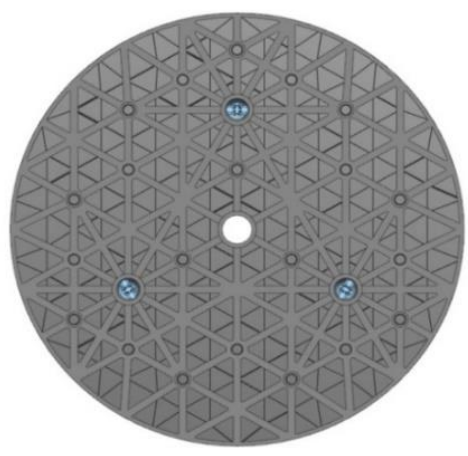

Figure 4. Ultra-lightweight design of the primary mirror and its support

After optimizing each mirror and the fuselage, the authors created a virtual prototype of the remote sensor. Table 5 lists the properties of the material of each component in the sensor.

Table 5. Material properties of the remote sensor

\begin{tabular}{cccccc}
\hline Components & Material & $\begin{array}{c}\text { Density } \boldsymbol{\rho} \\
\left(\mathbf{1 0}^{\mathbf{3}} \mathbf{k g} / \mathbf{m}^{\mathbf{3}}\right)\end{array}$ & $\begin{array}{c}\text { Elastic modulus E } \\
(\mathbf{M P a})\end{array}$ & $\begin{array}{c}\text { Poisson's } \\
\text { ratio } \boldsymbol{\mu}\end{array}$ & $\begin{array}{c}\text { Coefficient of linear expansion } \\
\boldsymbol{\alpha}\left(\mathbf{1 0}^{-6} /{ }^{\circ} \mathbf{C}\right)\end{array}$ \\
\hline $\begin{array}{c}\text { Primary mirror, Secondary } \\
\text { mirror, Tertiary mirror }\end{array}$ & $\mathrm{SiC}$ & 3.05 & 330000 & 0.25 & 2.3 \\
Mirror connector & Invar & 8.10 & 141000 & 0.25 & 2.0 \\
Flexible support & Titanium alloy & 4.44 & 114000 & 0.29 & 8.9 \\
Fuselage & Carbon fiber & 1.56 & 100000 & 0.30 & 0.3 \\
\hline
\end{tabular}

To approximate the actual structure, each component of the sensor was simulated by a solid model, and meshed into tennode tetrahedral (TET10) elements. The grid thickness was adjusted based on the requirements on each component. Besides, the components were constrained according to the designed interface. Under the constraints of public node connection, the established FEM contains 285,505 nodes (Figure 5).

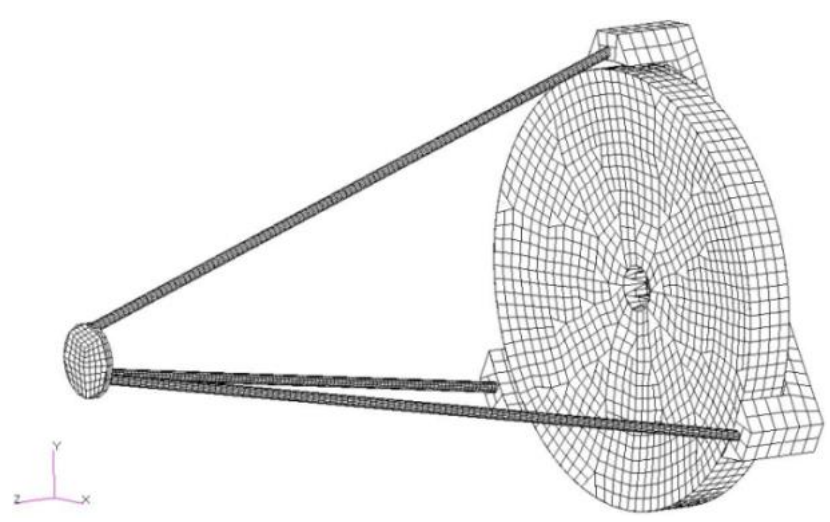

Figure 5. FEM of the remote sensor

\subsection{Gravity deformation of the remote sensor}

Because of the ultra-lightweight design, each mirror was greatly affected by gravity deformation. Then, a static analysis was performed under X-direction gravity, aiming to disclose how gravity affects the pose accuracy of mirrors, as the remote sensor is being fabricated and detected on the ground.

During sensor fabrication, the gravity deformation of each mirror is steady in the $\mathrm{X}$ direciton. Thus, the deformation of each mirror in the remote sensor was obtained through finiteelment analysis under the gravity of this direction. Table 6 lists the pose misalignment of each mirror under $\mathrm{X}$-direction gravity.

As shown in Table 6, when the sensor was fabricated and detected on the ground, the gravity-induced rigid body displacement of primary and secondary mirrors in the $\mathrm{X}$ direction surpassed the optical tolerance limit. Meanwhile, the pose angle of the primary mirror around the $\mathrm{Y}$ axis, and that of the secondary mirror around the $\mathrm{Y}$ axis also exceeded the tolerance limit. The gravity deformation of the mirrors cannot be further reduced through structural optimization.

The FEM analysis reveals that the rigid body displacement and pose angle of primary and secondary mirrors exceeded the tolerance limit under gravity. Therefore, it is necessary to estimate the misalignment-induced aberration of the optical system induced by gravity deformation. 
Table 6. Pose misalignment of each mirror under X-direction gravity

\begin{tabular}{ccccc}
\hline \multicolumn{2}{c}{ Gravity deformation } & $\begin{array}{c}\text { Primary } \\
\text { mirror }\end{array}$ & $\begin{array}{c}\text { Secondary } \\
\text { mirror }\end{array}$ & $\begin{array}{c}\text { Tertiary } \\
\text { mirror }\end{array}$ \\
\hline Rigid body & $\Delta \mathrm{X}$ & 25.6 & 36.2 & 6.4 \\
displacement & $\Delta \mathrm{Y}$ & -2.6 & -4.7 & -2.7 \\
$(\mu m)$ & $\Delta \mathrm{Z}$ & 0.9 & 1.0 & -0.2 \\
Mirror surface & $\theta_{\mathrm{X}}$ & 0 & -0.3 & -0.2 \\
tilt & $\theta_{\mathrm{Y}}$ & 2.7 & 3.6 & 1.4 \\
$(")$ & $\theta_{\mathrm{Z}}$ & 0.9 & 1.2 & 0.5 \\
\hline
\end{tabular}

\section{CORRECTION CAPABILITY OF SECONDARY MIRROR POSE ADJUSTMENT}

\subsection{Design of secondary mirror pose adjustment}

In the optical system, the secondary mirror is supported by the carbon fiber spider structure, which was selected for the purpose of ultra-lightweight design. Under gravity, the long cantilever of the support can easily cause rigid body displacement and pose angle deflection of the secondary mirror. Thus, it is very important to adjust the pose misalignment of this mirror.

Through the optical design and analysis, it was found that the secondary mirror serves as an intermediary between the primary and tertiary mirrors. This means the secondary mirror could act as a correction link: the adjustment of its pose could effectively compensate for the pose misalignments of primary and tertiary mirrors $[17,18]$.

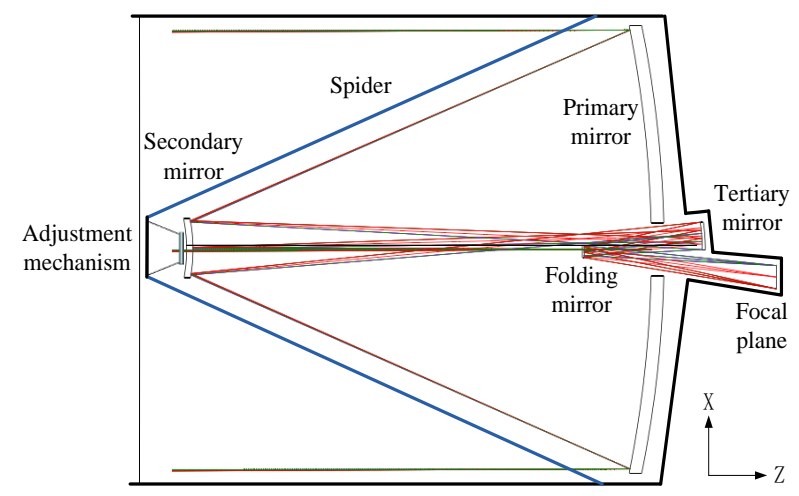

Figure 6. Design of secondary mirror pose adjustment

For the 5DOF pose adjustment of the secondary mirror, a driving mechanism was designed with high-precision positioning. The secondary mirror was mounted on the driving mechanism, and its pose was adjusted by changing the multiDOF movements of the driving mechanism. The overall adjustment design is explained in Figure 6.

\subsection{Correction effect on gravity deformation}

Because the pose misalignments of the primary and secondary mirrors surpassed the tolerance limits, the misalignment-induced aberration should be corrected by adjusting the pose of the secondary mirror $[19,20]$. Here, the capability of the secondary mirror pose adjustment to correct the gravity deformation of the optical system is simulated.

Table 7. Correction effect of the secondary mirror pose adjustment on gravity deformation

\begin{tabular}{|c|c|c|c|c|c|c|c|c|c|c|c|c|}
\hline \multirow{3}{*}{ Mirror } & \multicolumn{5}{|c|}{ Gravity deformation } & \multirow{3}{*}{$\begin{array}{c}\text { Post-focusing } \\
\text { aberration (RMS) }\end{array}$} & \multicolumn{5}{|c|}{ Secondary mirror pose adjustment } & \multirow{3}{*}{$\begin{array}{l}\text { Post-correction } \\
\text { aberration } \\
\text { (RMS) }\end{array}$} \\
\hline & Dx & Dy & $\mathrm{Dz}$ & Tx & $\mathrm{Ty}$ & & Dx & Dy & $\mathrm{Dz}$ & $\mathrm{Tx}$ & Ty & \\
\hline & \multicolumn{3}{|c|}{$(\mu \mathrm{m})$} & \multicolumn{2}{|c|}{$\left({ }^{\prime \prime}\right)$} & & & $(\mu m)$ & & & (') & \\
\hline $\begin{array}{l}\text { Primary } \\
\text { mirror }\end{array}$ & 25.6 & -2.6 & 0.9 & 0 & 2.6 & & & & & & & \\
\hline $\begin{array}{l}\text { Secondary } \\
\text { mirror }\end{array}$ & 36.2 & -4.7 & 1.0 & -0.3 & 2.8 & $\begin{array}{l}0.078 \sim \\
0.095 \lambda\end{array}$ & 27.8 & 2.0 & 11.8 & 0 & 0 & $\begin{array}{l}0.012 \sim \\
0.022 \lambda\end{array}$ \\
\hline $\begin{array}{c}\text { Tertiary } \\
\text { mirror }\end{array}$ & 6.4 & -2.7 & -0.2 & -0.2 & 0.6 & & & & & & & \\
\hline
\end{tabular}

According to the simulation results (Table 7), when the sensor was fabricated and detected on the ground, the misalignments of the mirrors induced by gravity deformation cannot be effectively compensated for through focusing alone. After adjusting the pose of secondary mirror, the gravity deformation of the optical system was corrected well. The post-correction aberration was close to the theoretical value of the optical design, marking the success of fabrication and detection of the large aperture remote sensor on the ground.

\subsection{Correction effect on in-orbit misalignment}

Despite its good imaging effect on the ground, the large aperture remote sensor will encounter vibration, shock, and overload in the launch process. These mechanical conditions will cause stress release, and degrade the performance of local assemblies in the opto-mechanical structure. After entering into the orbit, the remote sensor will face an environment of microgravity. The change of gravity field will cause a resurgence of gravity deformation of the opto-mechanical structure. Moreover, the poses of the mirrors will change through the folding and unfolding processes, resulting in misalignment and wave aberration in the optical system.

The impact of gravity rebound can be determined through simulation analysis, but the stress release and local assembly degradation can only be estimated empirically. Considering the effect of gravity in all directions, the worst-case in-orbit pose misalignment was set as 10 times the maximum pose misalignment of each mirror.

Under the in-orbit scenario, the misalignment-induced aberration of the remote sensor was measured by the wave front measurement module. The correction effect in Table 6 was selected to derive the pose adjustment amount of the secondary mirror in orbit, laying the basis for correcting the misalignment caused by gravity rebound in orbit.

Furthermore, the misalignment induced by vibration, shock, and overload was finetuned in a similar manner. The worstcase in-orbit pose misalignment of the remote sensor was estimated, and 100 misalignments were randomly sampled by the Monte-Carlo (MC) method from the optical system. Then, the correction effect on in-orbit misalignment was simulated. 


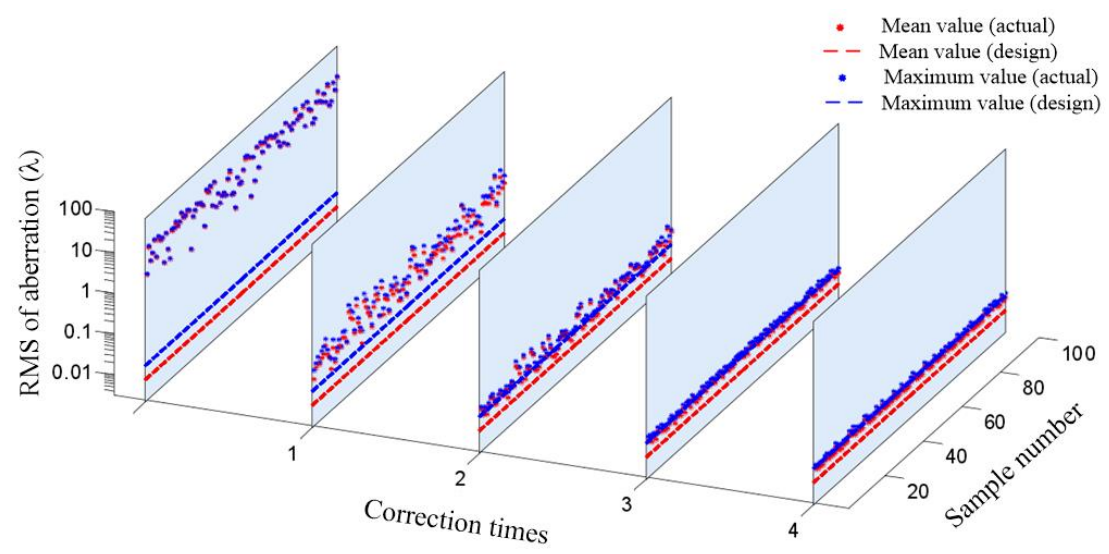

Figure 7. Correction effect of the secondary mirror pose adjustment on misalignments in orbit

As shown in Figure 7, the correction effect did not deteriorate with the increase of the misalignment amount. After the first and second corrections, the aberration of each sample decreased significantly. In the next two corrections, however, the aberration of each sample declined slightly. Through three corrections, the mean and maximum RMSs of wave aberration were both reduced to less than $0.029 \lambda$. Hence, the optical system basically reaches the designed level of aberration after the correction. Then, the remote sensor can realize high-accuracy imaging in orbit. The M-C simulation confirmed that the random misalignments of the optical system have no obvious impact over the correction effect on in-orbit misalignment, indicating that the proposed design for secondary mirror pose adjustment is adaptable to the uncertain misalignment of the remote sensor in orbit.

\section{CONCLUSIONS}

The ultra-lightweight design has amplified the effect of gravity on large aperture remote sensor. However, it is difficult to implement a gravity unloading mechanism on such a sensor. To overcome the difficulty, this paper puts forward a novel design for secondary mirror pose adjustment. This design is a necessary means to offset the gravity deformation of ultralightweight large aperture remote sensor.

Through simulation analysis, the pose adjustment design was proved capable of correcting the aberration induced by gravity deformation, when the sensor is being fabricated and detected on the ground. Besides, the aberration caused by gravity release and other factors in the launch process could also be corrected by the proposed design. As for the uncertain misalignments in orbit, the worst-case misalignment was estimated, and multiple misalignments were sampled by the MC method for simulation. The simulation results show that, after three corrections, the misalignments were reduced to less than $0.029 \lambda$, indicating that the proposed design for secondary mirror pose adjustment is adaptable to the uncertain in-orbit misalignments of the remote sensor. The proposed design greatly reduces the difficulty and cost of the large aperture remote sensor in gravity unloading, and effectively improves the sensor's imaging quality in orbit.

\section{ACKNOWLEDGMENT}

This work was funded by National Key Basic Research and Development Plan Project of China (Grant No:
2016YFB0500100) and National Natural Science Foundation of China (Grant No: 11873007).

\section{REFERENCES}

[1] Chang, J., Weng, Z.C., Jiang, H.L. (2003). Design on three-reflective-mirror system used in space. Acta Optica Sinica, 23(2): 216-219. https://doi.org/10.3321/j.issn:0253-2239.2003.02.018

[2] Guo, J., He, X. (2008). Support structure design of primary mirror for large-aperture space remote sensing camera. Optics and Precision Engineering, 16(9): 16421647 https://doi.org/10.3321/j.issn:1004924X.2008.09.013

[3] Yan, Y., Jia, J.Q., Jin, G. (2008). Design of new lightweight large-aperture space mirror support. Optics and Precision Engineering, 16(8): 1533-1539. https://doi.org/10.3321/j.issn:1004-924X.2008.08.031

[4] Li, Z.L., Xue, D.L., Zhang, X.J. (2008). Opticalmechanical structure design of long focal length and large field of view optical system. Optics and Precision Engineering, 16(12): 2485-2490. https://doi.org/10.3321/j.issn:1004-924X.2008.12.027

[5] John, W. (1999). Figoski. Alignment and test results of the quick bird telescope using the ball optical system test facility. International Society for Optics and Photonics, 3785: 99-108. https://doi.org/10.1117/12.367607

[6] Abdulkadyrov, M.A., Patrikeev, A.P., Belousov, S.P. (2008). M2 secondary mirror manufacturing for VISTA project. International Society for Optics and Photonics, 7018: B1-B9. https://doi.org/10.1117/12.789013

[7] Geijo, E.M., Casalta, J.M., Canchado, M., San Andrés, M., Brú, R., García, H., Jeffers, P. (2006). VISTA secondary mirror drives performance and test results. In Optomechanical Technologies for Astronomy, 6273: 3801-3810. https://doi.org/10.1117/12.669949

[8] Huang, Y.F., Lin, L., Cao, Y.H. (2006). Computer-aided alignment for space telescope optical system. International Symposium on Advanced Optical Manufacturing and Testing Technologies: Advanced Optical Manufacturing Technologies, 6149: 61490P. https://doi.org/10.1117/12.674212

[9] Seery, B.D. (2003). Next generation space telescope (NGST): Hubble's scientific and technological successor. In IR Space Telescopes and Instruments. 4850: 170-178. https://doi.org/10.1117/12.461780

[10] Lightsey, P.A., Chaney, D., Gallagher, B., Brown, B., 
Smith, K., Lewis, J., Siegel, N. (2010). Optical performance for the actively controlled James Webb Space Telescope. In Space Telescopes and Instrumentation 2010: Optical, Infrared, and Millimeter Wave, 7731(1):

52-56.

https://doi.org/10.1117/12.859091

[11] Chheta, Y.R., Joshi, R.M., Gotewal, K.K., ManoahStephen, M. (2017). A review on passive gravity compensation. In 2017 International conference of Electronics, Communication and Aerospace Technology (ICECA), 1 : 184-189. https://doi.org/10.1109/ICECA.2017.8203668

[12] Jia, J., Jia, Y.M., Sun, S.H. Preliminary design and development of an active suspension gravity compensation system for ground verification. Mechanism and Machine Theory, 128: 492507.https://doi.org/10.1016/j.mechmachtheory.2018.06. 018

[13] Jiménez, A., Morante, E., Viera, T., Núñez, M., Reyes, M. (2010). Design of a prototype position actuator for the primary mirror segments of the European Extremely Large Telescope. In Ground-based and Airborne Telescopes $\quad$ III, 7733: 773354. https://doi.org/10.1117/12.857158

[14] Li, L., Zhao, Y. (2016). A gravity unloading method of on-ground alignment for large aperture remote sensor. Spacecraft Recovery \& Remote Sensing, 37(5): 69-76. https://doi.org/10.3969/j.issn.1009-8518.2016.05.008
[15] Schmid, T., Thompson, K.P., Rolland, J.P. (2010). Misalignment-induced nodal aberration fields in twomirror astronomical telescope. Applied Optics, 49(16): D131-D144. https://doi.org/10.1364/AO.49.00D131

[16] Fuerschbach, K., Rolland, J.P., Thompson, K.P. (2014). Theory of aberration fields for general optical systems with freeform surfaces. Optics Express, 22(22): 2658526606. https://doi.org/10.1364/OE.22.026585

[17] Lee, H., Dalton, G.B., Tosh, I.A., Kim, S.W. (2008). Computer-guided alignment III: Description of interelement alignment effect in circular-pupil optical systems. Optics Express, 16(15): 10992-11006. https://doi.org/10.1364/oe.16.010992

[18] Thompson, K.P., Schmid, T., Cakmakci, O., Rolland, J.P. (2009). Real-ray-based method for locating individual surface aberration field centers in imaging optical systems without rotational symmetry. J. Opt. Soc. Am. A, 26(6): $1503-1517$ https://doi.org/10.1364/JOSAA.26.001503

[19] Kim, S., Yang, H.S., Lee, Y.W., Kim, S.W. (2007). Merit function regression method for efficient alignment control of two-mirror optical systems. Optics Express, 15(8):

5059-5068. https://doi.org/10.1364/OE.15.005059

[20] Gu, Z., Yan, C., Wang, Y. (2015). Alignment of a threemirror anastigmatic telescope using nodal aberration theory. Optics Express, 23(19): 25182-25201. http://doi.org/10.1364/oe.23.025182 